\title{
Real-Time State Estimation in the Cameroon Power System
}

\author{
Emmanuel Tanyi and Edwin Mbinkar \\ Automation and Control Laboratory, National Advanced School of Engineering, University of Yaounde 1, B.P. 8390 Yaounde, Cameroon
}

Correspondence should be addressed to Edwin Mbinkar; mbinkaren@yahoo.fr

Received 14 May 2013; Accepted 30 June 2013

Academic Editors: A. R. Beig, H. Ghezel-Ayagh, and D. Zhang

Copyright (C) 2013 E. Tanyi and E. Mbinkar. This is an open access article distributed under the Creative Commons Attribution License, which permits unrestricted use, distribution, and reproduction in any medium, provided the original work is properly cited.

\begin{abstract}
An important tool for the energy management system (EMS) is state estimation. Based on measurements taken throughout the network, state estimation gives an estimation of the state variables of the power system while checking that these estimates are consistent with the measurements. Currently, in the Cameroon power system, state estimates have been provided by ad hoc supervisory control and data acquisition (SCADA) systems. A disadvantage is that the measurements are not synchronised, which means that state estimation is not very precise during dynamic phenomena in the network. In this paper, real-time phasor measurement units (PMUs) that provide synchronised phasor measurements are proposed for integration into the power system. This approach addresses two important issues associated with the power system state estimation, namely, that of measurement accuracy and that of optimization of the number of measurement sites, their location, and the importance given to their measurements on the dynamic state estimation.
\end{abstract}

\section{Introduction}

The electricity sector in Cameroon has been undergoing gradual deregulation in the last few years. AES-SONEL, which took over the generation, transmission, and distribution of electricity in the country in 2001, disposes of a total installed capacity of $933 \mathrm{MW}$ (721 MW from hydroelectricity sources and 212 from thermal sources including $24 \mathrm{MW}$ of isolated capacity) [1]. The transportation network has $480 \mathrm{~km}$ of $225 \mathrm{kV}$ lines, $337 \mathrm{~km}$ of $110 \mathrm{kV}$ lines, and $1064 \mathrm{~km}$ of $90 \mathrm{kV}$ lines, with 24 sub-stations.

The need for better utilization of power system facilities has increased significantly in the last decade and will continue, as the electricity market is becoming more complex and competitive. More real-time data from substations and generating plants is required to support SCADA (supervisory control and data acquisition) and EMS (energy management system) in utility control centres. There has been a significant increase in interest in planning, operating, equipment, and maintenance personnel for real-time events and archived data. This wide interest demonstrates the need for the use of real-time state estimators.
The system control centre in Mangombe is responsible for the load dispatch and reliability of the main $225 \mathrm{kV}$ grid. Tanyi and Mbinkar have proposed that four area control centres can be located throughout the regions covering the southern interconnected grid (SIG) to be responsible for the operation and maintenance procedures on the transmission, subtransmission, and distribution networks in their respective areas [2]. There are presently a few telemetry systems, and all generating plants and major substations have separate remote terminal units (RTUs) and/or remote supervisory and telemetry systems reporting to both the system and area control centres.

Almost all the RTUs and older remote supervisory and telemetry systems connect to the control centres over dedicated telecommunication circuits on a one-on-one basis usually over telecommunication media owned by Cameroon Telecommunications (CAMTEL). CAMTEL has an extensive microwave telecommunication network for protective relaying, remote supervisory control, and operational voice requirements. There are also many power line carrier telecommunication links. Both the microwave and the power line carrier equipment are moving towards digital technology 
with the deregulation process. Some stations are not accessible by the telephone common carrier, and most of these use point-to-multipoint cellular radio communications.

State estimation forms an important part of the modern day control centre and substation automation functions. State estimation calculates the voltage magnitudes and angles at all the buses of the power system. The accuracy of the state estimation output is very crucial, as it forms the basis for the EMS functions like security analysis, voltage stability analysis, optimal load dispatch, and so forth. The power system is not a static system, but it changes with time, though very slowly. This is essentially due to the continuous variation of loads. Once the loads change, the generation has to keep up to those changes, and this triggers a change in the generations. This in turn leads to a change in flows and injections at all the buses of a power system. These dynamic changes in the power system are difficult to be captured by the traditional static state estimation (SSE) in place.

The accuracy of the state estimation depends upon the quality of the communication links and the measurements used for the state estimation. Traditionally, line flows, injections, and voltage measurements were the most commonly used measurements for state estimation. In the 1980s, a new measuring instrument called the phasor measurement unit (PMU) was developed [3]. The specificity of the device is that it can directly measure the voltage phasors and current phasors at a given bus. In other words, it can directly measure voltage magnitude and its angle, which forms the state vector. Hence, if all the buses were to have PMUs installed and their measurements were transmitted to the control centre, we can have a complete real-time measurement of the state vector of the power system, instead of estimation. But due to cost considerations, PMUs cannot be installed at all the locations of a power system. Hence, the state estimators need to process the normal SCADA data along with the PMU data to obtain a good estimate of the states of the power system at a given instance.

In this paper, a measurement placement algorithm for the PMUs is proposed. The proposed algorithm is applied to find the positions of measurement placement in the southern interconnected grid (SIG) of the Cameroon power system. The number of PMU measurements is also very important for the accuracy of the state estimation. The results obtained are based on simulations carried out using an evaluation version of PowerWorld software.

\section{Mathematical Formulation}

2.1. Measurement Models. The information model used in power system state estimation is represented by the equation [4]

$$
z=h(x)+w
$$

where $z$ is a $(m * 1)$ measurement vector obtained from PMUs, $x$ is a $(n * 1)$ true state vector, $h(\cdot)$ is a $(m * 1)$ vector of nonlinear functions, $w$ is a $(m * 1)$ measurement error vector, $m$ is the number of measurements, and $n$ is the number of state variables. The usual choice for state variables is the bus voltage phase angles and magnitudes, while the measurements are active and reactive power flows, node injections and voltage magnitudes.

As in load flow calculations, it has been found that state estimation algorithms based on decoupled versions of (1) behave adequately for the usual power networks [4]. Consequently, the following decoupled model is usually adopted:

$$
\begin{aligned}
& z_{p}=h_{p}(\theta, v)+w_{p}, \\
& z_{q}=h_{q}(\theta, v)+w_{q},
\end{aligned}
$$

where $\theta\left(n_{\theta} * 1\right)$ and $v\left(n_{v} * 1\right)$ are the vectors of true voltage magnitudes and phase angles and $p$ and $q$ are subscripts indicating partitions of vectors and matrices corresponding to active and reactive measurements, respectively; $n_{\theta}=N-1$, $n_{v}=N$, and $N$ is the number of network nodes.

2.2. Problem Formulation. The state estimation problem of a power system can be considered as the following weighted least squares (WLS) method optimization problem [5]:

$$
\operatorname{Min} J(x)=[z-h(x)]^{T}[R]^{-1}[z-h(x)],
$$

where $[R]$ is called a measurement covariance matrix. Usually, the distribution of each measurement error is assumed to be Gaussian noise with zero mean and independent; thus, $[R]$ becomes a diagonal matrix [6]. These weights are chosen as proportional to the accuracy of measurement. Normally, the covariance of the measurements is unknown and often assumed to be an identity matrix since the same instrumentation is used to obtain them. The solution of (3), in WLS sense, is obtained by

$$
[h]^{T}[R]^{-1}[h](x)=[h]^{T}[R]^{-1}[h] z .
$$

In this paper, the voltage phasors at all busbars are chosen as state variables, since they allow the branch currents, shunt currents, and currents injected into the busbar to be determined [5]. The measured values are the busbar voltage phasors, the injection current phasors, and the line current phasors. Based on these measured values, all possible relations of the estimated state variable vector $v$ and the measurement vector $z$ in the presence of measurement error $w$ can be expressed as

$$
\left[\begin{array}{c}
z_{V} \\
z_{I} \\
z_{L}
\end{array}\right]=\left[\begin{array}{cc}
{[I]} & {[0]} \\
{\left[Y_{I M}\right]} & {\left[Y_{I C}\right]} \\
{\left[Y_{L M}\right]} & {\left[Y_{L C}\right]}
\end{array}\right]\left[\begin{array}{c}
v_{M} \\
v_{C}
\end{array}\right]+\left[\begin{array}{c}
w_{V} \\
w_{I} \\
w_{L}
\end{array}\right],
$$

where $[I]$ is an identity matrix, $z_{V}, z_{I}$, and $z_{L}$ are the measured values of the busbar voltage, the injection current, and the line current subvectors, respectively, $Y_{I M}$ and $Y_{I C}$ are the bus admittance submatrices of the measured and the calculated busbar voltage related to $z_{I}$, respectively, $Y_{L M}$ and $Y_{L C}$ are the bus admittance submatrices of the measured and the calculated busbar voltage related to $z_{L}$, respectively, $v_{M}$ and $v_{C}$ are the measured and the calculated busbar voltage subvectors, respectively, and $w_{V}, w_{I}$, and $w_{L}$ are the corresponding measurement error or noise subvectors. 


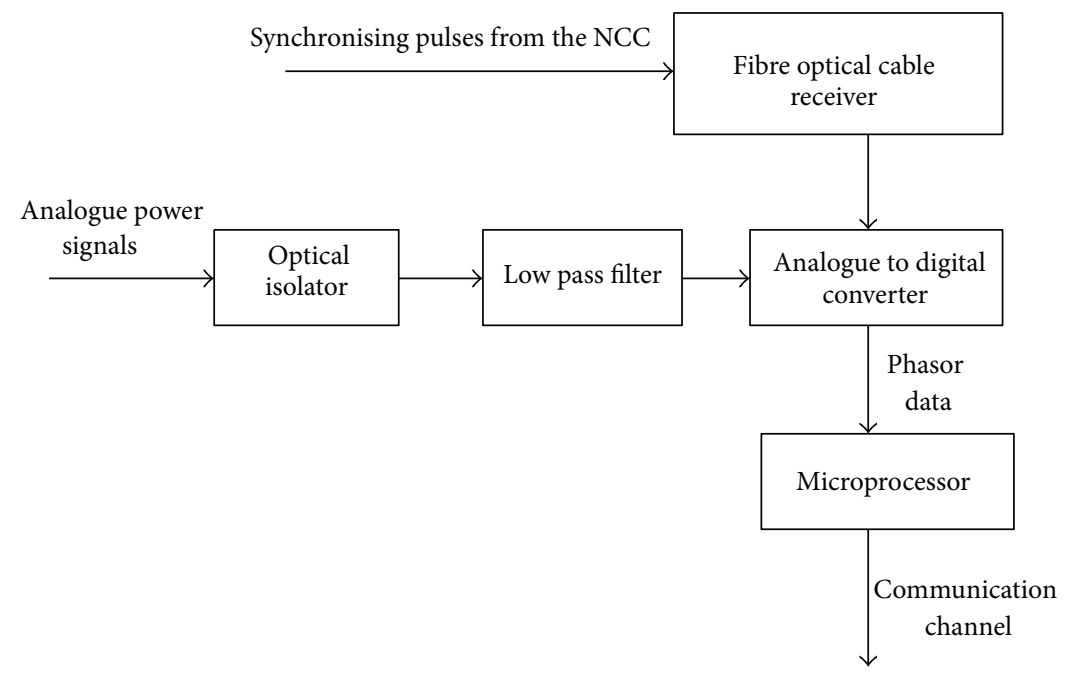

FIgURE 1: Basic block diagram of a PMU.

Equation (5) indicates all possible relationships between the measured values and the estimated state variables [5]. In practice, the measuring devices will not be placed at all possible positions for economic reasons. Thus, it is necessary to find an appropriate measurement placement position set such that all state variables can still be estimated with a minimum number of measuring devices.

\section{Operating Principles of a PMU}

As shown in Figure 1, the analogue power signal is converted into digital data by the analog to digital converter. For example, if we want to measure the voltage of the signal, then samples are taken for each cycle of the waveform, and then the fundamental frequency component is calculated as follows using discrete Fourier transform (DFT) [7]:

$$
X=\frac{\sqrt{2}}{N} \sum_{k=1}^{N} x_{k} e^{-j k(2 \pi / N)},
$$

where $N$ is the total number of samples in one period, $X$ is the phasor, and $x_{k}$ are the waveform samples.

From the above expression, we can see that the phasor information is dependent on the number of samples of the waveform and will give a correct representation of the fundamental frequency component even in the presence of transient signals. The samples are taken over one cycle or over multiple cycles of the power signal.

Data windowing is used to smoothen the frequency spectrum and minimise overshoot of the signal caused due to discontinuities of the signal in the time domain [7].

The optical isolator shields the PMU system from the very high bus voltages. The unit is composed of a number of phasors that capture measurements of analogue voltage, current waveform, and the line frequency. After that, the phasor measurements are digitized by an analogue to digital converter and stamped with the creation time provided by a clock at the network control centre (NCC). The clock pulses are typically supplied by GPS, but optical fibre cables have proven to be better [5]. These pulses are used for synchronization of multiple PMUs with a precision of maximum 1 microsecond difference. Afterwards, the data is transferred to a phasor data concentrator, where data with the same creation time are encapsulated in one packet and then transmitted as a single stream to the phasor data server which can be a PSGuard [2] or traditional SCADA. PMU provides a dynamic system observation of the network, because the measurements are taken with a high sampling rate from geographically distant locations, and they are then grouped together according to the time stamp provided. Phasor measurement unit transmits samples in different sizes. The sample size depends on the number of phasors in a unit. Samples and packets have the same meaning when talking about PMU transfer rate. The required transfer rate differs from a $50 \mathrm{~Hz}$ system to a $60 \mathrm{~Hz}$ system. For example, a $60 \mathrm{~Hz}$ system has a rate of up to 60 samples per second, while the $50 \mathrm{~Hz}$ one has up to 50 samples per second [8].

The PMUs measure voltage, current, and frequency phasors using the discrete Fourier transform (DFT) and can detect transients or surges within milliseconds of their occurrence [9].

PMUs use the IEEE 1344 data format for communication with the central monitoring station. In addition to the propagation delay of the particular link, the message format of the PMU and data rate of the link determine the communication delay in the system. Furthermore, there is also a processing delay due primarily to the window size of the DFT. As shown in Figure 2, PSGuard system utilizes phasors which are measured by PMUs resulting in an accuracy of $<1 \mu \mathrm{s}$ [9]. A communication system links PMUs, which are located in substations to the PSGuard system for data transmission. Data exchange can be established between PSGuard and other control and protection systems to allow for optimum data sharing and control actions. This calls for 


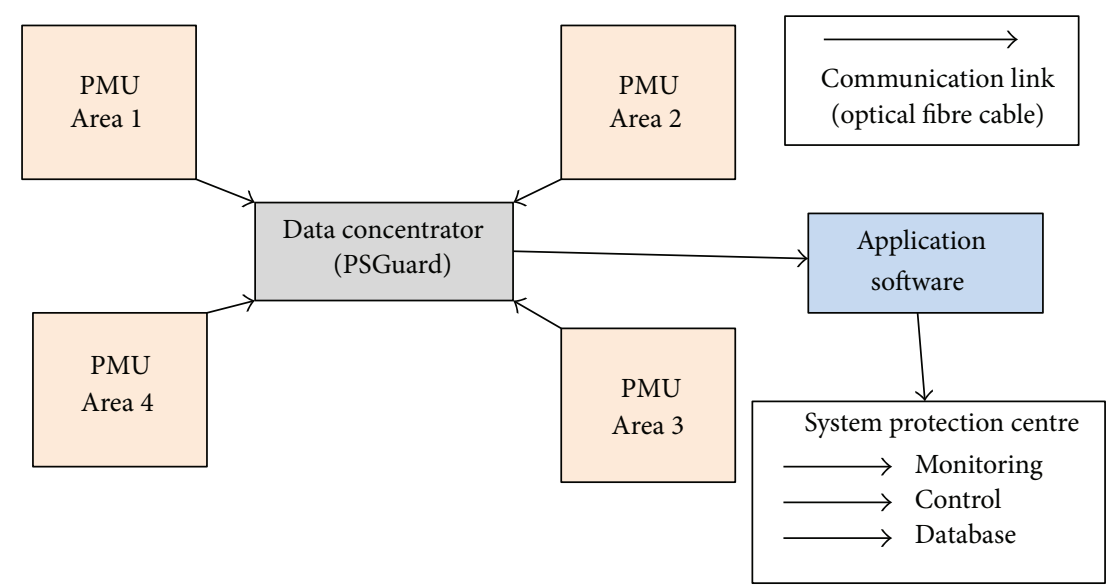

Figure 2: Placement of PMUs for measurements.

an efficient communication network for the SIG. There are already several projects in progress for the installation of fibre optic cables [10].

\section{Measurement Placement Algorithm}

The proposed measurement placement algorithm for the PMUs was described using three IEEE test systems [5] and is now being applied to the SIG. It is assumed that the power network is decomposed into $r$ areas [4]. The areas are connected through boundary buses which belong simultaneously to both adjacent areas.

4.1. Decomposition Technique. It is assumed that the entire network under consideration is decomposed into $r$ nonoverlapping observable areas. Each subnetwork consists of internal buses, which are adjacent to branches belonging only to this subnetwork, and boundary buses. These buses are connected to other areas by tie lines. For example, decomposition of a network into four nonoverlapping areas is shown in Figure 3. To obtain decoupled state estimation equations of these areas, constraints of measurement placement are as follows.

(i) There is no injection current measurement at the boundary bus.

(ii) There is no line current measurement on the tie line.

(iii) Each subnetwork has at least one busbar voltage measuring device.

Based on the previous conditions, parameters of the tielines do not appear in the estimation equations, and the measurement model of the power system state estimation can be written as

$$
\left[\begin{array}{c}
z_{1} \\
z_{2} \\
\vdots \\
z_{r}
\end{array}\right]=\left[\begin{array}{llll}
{\left[h_{1}\right]} & & & {[0]} \\
& {\left[h_{2}\right]} & & \\
& & \ddots & \\
{[0]} & & & {\left[h_{r}\right]}
\end{array}\right]\left[\begin{array}{c}
v_{1} \\
v_{2} \\
\vdots \\
v_{r}
\end{array}\right]+\left[\begin{array}{c}
w_{1} \\
w_{2} \\
\vdots \\
w_{r}
\end{array}\right],
$$

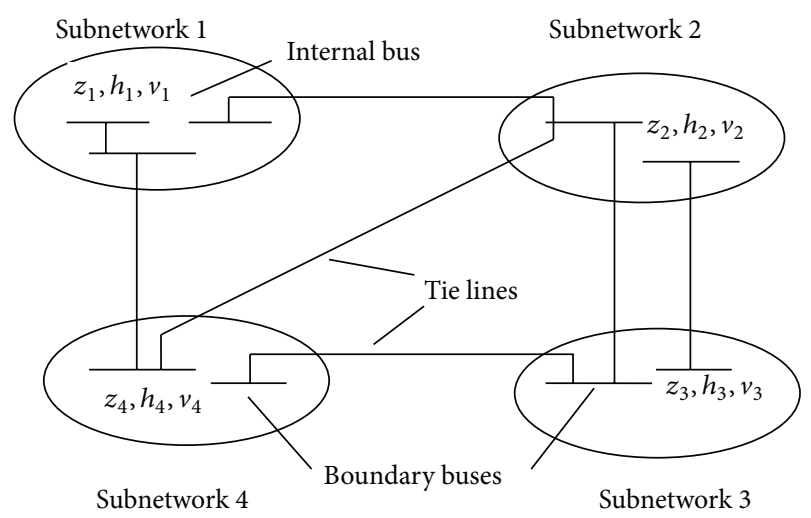

FIGURE 3: Decomposition of a network into four nonoverlapping sub-networks.

where $z_{r}, v_{r}$, and $h_{r}$ denote the measurement vector, the state variable vector, and the measurement matrix of the $i$ th subnetwork, respectively. $w_{r}$ represents the measurement error vector related to $z_{r}$. These $z_{r}, v_{r}, h_{r}$, and $w_{r}$ are formulated according to (5) for the $r$ th subnetwork.

The state variables of each sub-network can be estimated independently. Since the size of the sub-network is smaller, determining an optimal measurement placement can be performed with less computation effort using either the minimum condition number criteria [11] or other approaches. The optimal measurement placement of each sub-network is selected independently to ensure that the number of measurements is equal to the number of state variables. Thus, the measurement placement obtained from the minimum condition number criteria can be rearranged to minimize the number of placement sites by the following heuristic algorithm [11].

\subsection{Reduction of Placement Sites}

Step 1. Based on the placement position list obtained from the measurement placement algorithm, determine the bus where either an injection current or a busbar voltage measuring 


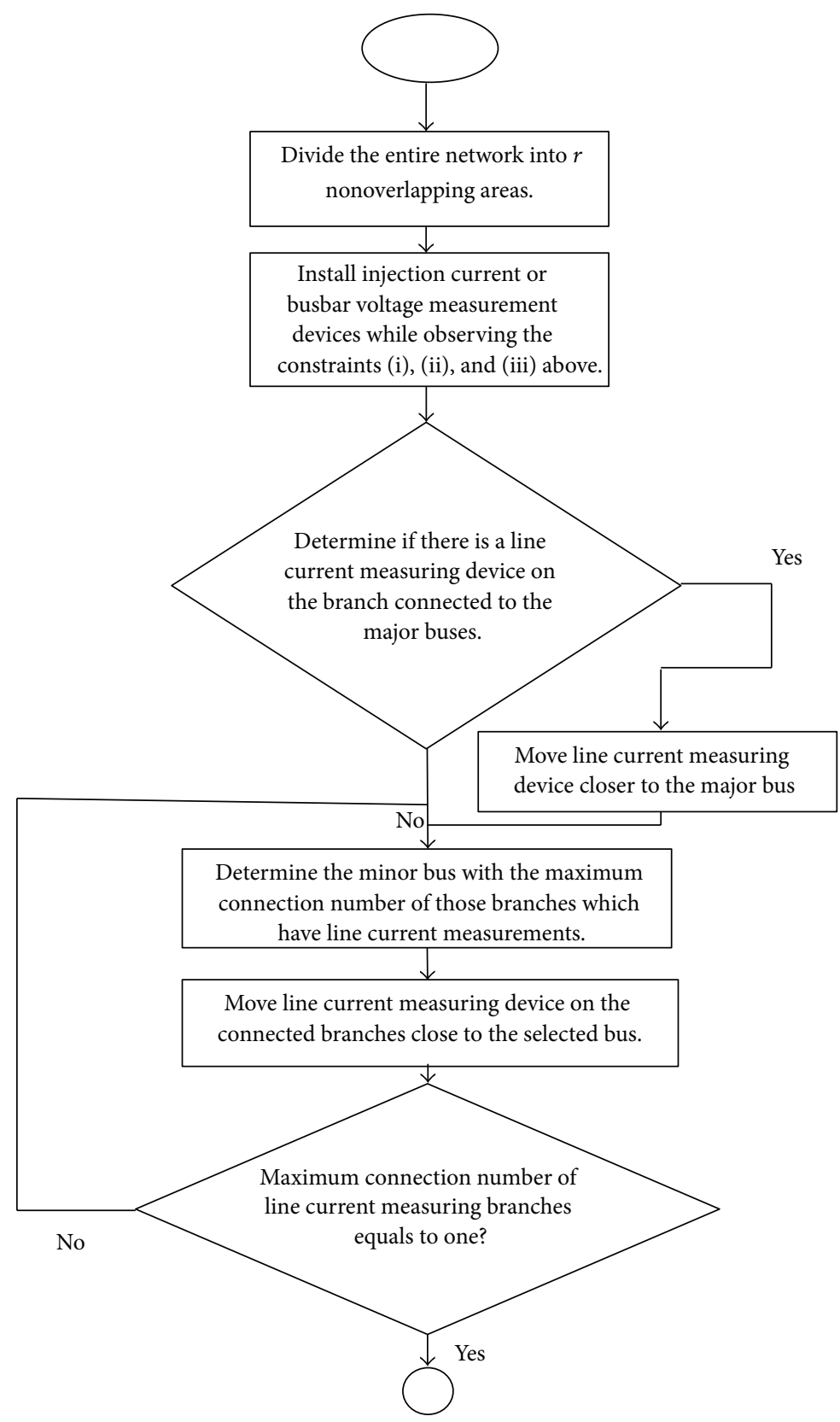

FIGURE 4: Flowchart for reduction of PMU placement sites.

device is installed. These buses are called major buses. Other buses are called minor buses.

Step 2. If there is a line current measuring device on the branch connected to the major buses, the device is moved close to the major buses.

Step 3. From the branches with line current measuring devices which are not connected to major buses, determine the minor bus with the maximum connection number of those branches which have line current measurements. Then, the line current measuring device on the connected branches is moved close to the selected bus. Note that this minor bus will not be considered again in the next iteration.

Step 4. Repeat Step 3 until the maximum connection number of line current measuring branches equals to one.

Figure 4 illustrates the algorithm for the reduction of placements sites with a flowchart.

\section{Illustration and Results}

The flowchart presented in Figure 4 is illustrated using the six-bus power system as shown in Figure 5, in which there are 

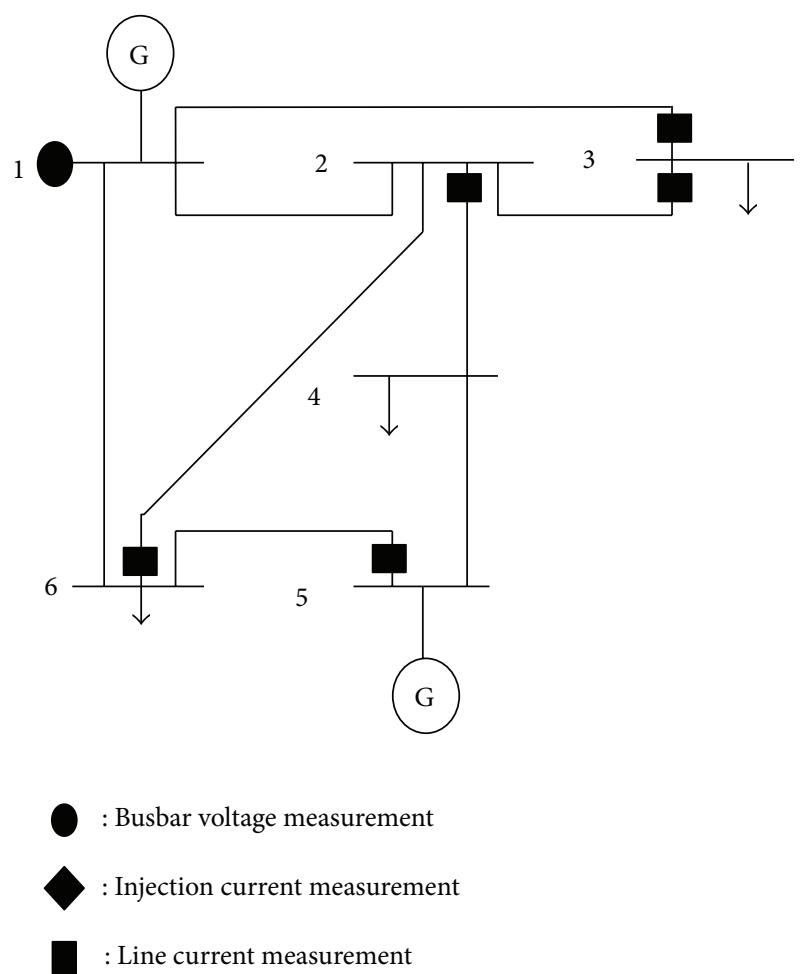

FIGURE 5: Six-bus power system with five PMU placement sites.

five placement sites and two generators [5], and then applied to the high voltage network $(225 \mathrm{kV} / 90 \mathrm{kV})$ of the SIG.

5.1. Six-Bus Power System. The six-bus power system with five PMU placement sites and two generators is shown in Figure 5.

Step 1. Determine the major buses and minor buses (see Table 1).

Step 2. Move devices close to major buses (see Table 2).

Step 3. We can see that bus number 2 has the maximum connection number of three, since it is connected to buses 3,4 , and 6 . Thus, the bus no. 2 will be chosen in this iteration. Then, the device is moved close to bus no. 2 .

Step 4. After moving the device, buses 5 and 6 have the connection number of one. Thus, the iteration procedure is stopped, and the placement positions of the six-bus power system become as shown in Figure 6. Notice that the number of placement sites reduces to three (see Table 3 ).

5.2. Application to the SIG. The SIG has two main generating stations located at Edea and Songloulou which are interconnected to the high voltage network using $225 \mathrm{kV}$ and $90 \mathrm{kV}$ transmission lines. There are five main substations-Bekoko, Logbaba, Mangombe, Oyomabang, and Nkongsamba [2]. Figure 7 shows the interconnection of the busbars of these substations with tie lines. The generator at Bekoko represents

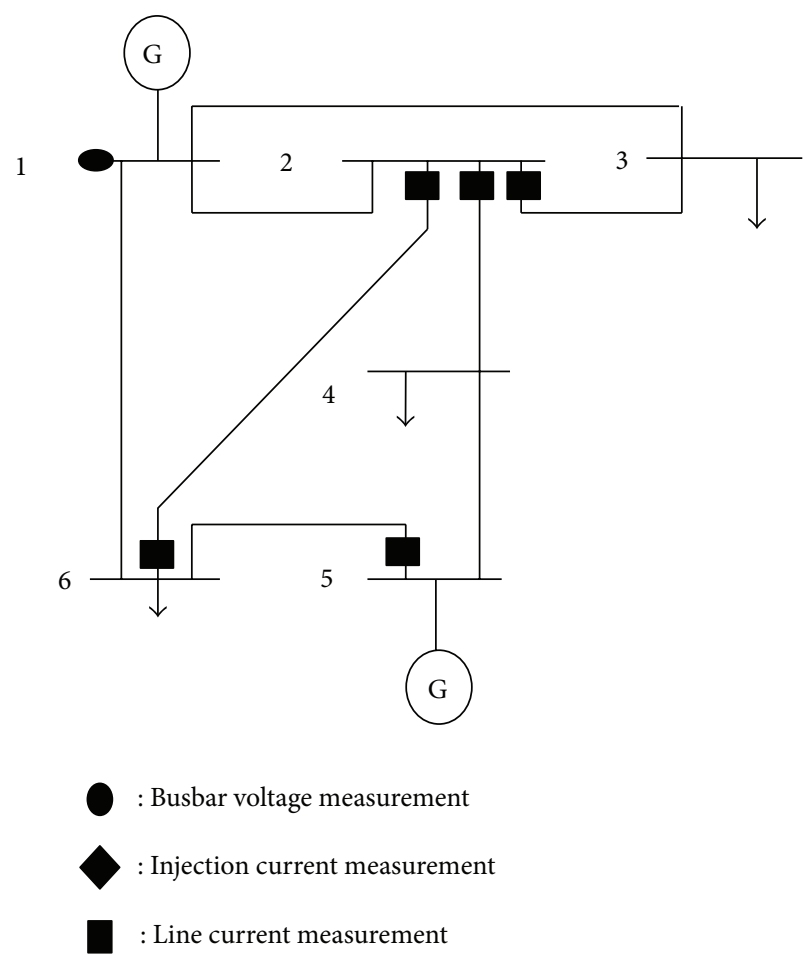

Figure 6: Optimal placement sites for PMUs in the six-bus power system.

TABLE 1

\begin{tabular}{lccc}
\hline From & To & Measurement type & Bus type \\
\hline 1 & - & Busbar voltage & Major \\
2 & 4 & Line current & Minor \\
3 & 1 & Line current & Minor \\
3 & 2 & Line current & Minor \\
5 & 6 & Line current & Minor \\
6 & 2 & Line current & Minor \\
\hline
\end{tabular}

TABLE 2

\begin{tabular}{lccc}
\hline From & To & Measurement type & Bus type \\
\hline 1 & - & Busbar voltage & Major \\
2 & 4 & Line current & Minor \\
1 & 3 & Line current & Minor \\
3 & 2 & Line current & Minor \\
5 & 6 & Line current & Minor \\
6 & 2 & Line current & Minor \\
\hline
\end{tabular}

the Limbe thermal plant. The entire network is divided into three areas.

The load dispatch centre is located at Mangombe and is responsible for monitoring and controlling the entire electrical network through regional control centres located at the other substations. The communication network between the different substations consists of optical fibre cables. The algorithm previously described is applied to the SIG to determine the optimal number and placement sites for the PMUs. 


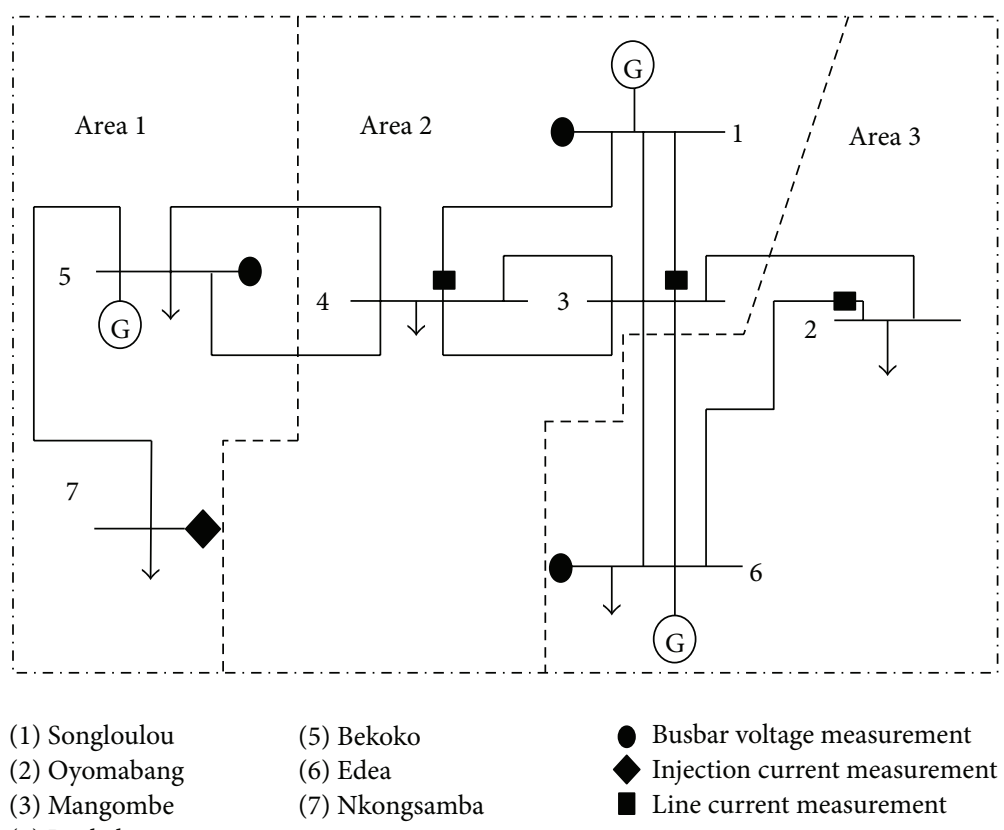

(4) Logbaba

FIGURE 7: Installation of measuring devices within the SIG.

TABLE 3

\begin{tabular}{lccc}
\hline From & To & Measurement type & Bus type \\
\hline 1 & - & Busbar voltage & Major \\
2 & 4 & Line current & Minor \\
1 & 3 & Line current & Minor \\
2 & 3 & Line current & Minor \\
5 & 6 & Line current & Minor \\
2 & 6 & Line current & Minor \\
\hline
\end{tabular}

TABLE 4

\begin{tabular}{lccc}
\hline From & To & Measurement type & Bus type \\
\hline 1 & - & Busbar voltage & Major \\
2 & 6 & Line current & Minor \\
3 & 1 & Line current & Minor \\
4 & 1 & Line current & Minor \\
5 & - & Busbar voltage & Major \\
6 & - & Busbar voltage & Major \\
7 & - & Injection current & Major \\
\hline
\end{tabular}

Step 1. Determine the major buses and minor buses (see Table 4).

Step 2. Move devices close to major buses (see Table 5).

Step 3. It is seen that bus no. 1 has the maximum connection number of two, since it is connected to buses 3 and 4 . But the devices are already moved to bus no. 1 . So, the algorithm ends.
TABLE 5

\begin{tabular}{lccc}
\hline From & To & Measurement type & Bus type \\
\hline 1 & - & Busbar voltage & Major \\
1 & 3 & Line current & Minor \\
1 & 4 & Line current & Minor \\
5 & - & Busbar voltage & Major \\
6 & 2 & Line current & Minor \\
6 & - & Busbar voltage & Minor \\
7 & - & Injection current & Major \\
\hline
\end{tabular}

The number of placement sites is consequently reduced from seven to four. These are located at Songloulou, Edea, Bekoko, and Nkongsamba.

5.3. Real-time Simulation of the SIG. A load flow analysis of the reduced network with an evaluation version of PowerWorld is seen in picture form in Figure 9. What stands out is that the line from Bekoko to Nkongsamba is heavily loaded beyond its dynamic and thermal limits. This makes the SIG unstable and unreliable, and the transmission losses must be high. There are parallel lines between the generating points and the major load centres in Douala and Yaounde. However, these parallel lines are of two different transmission voltages (90 and $225 \mathrm{kV}$ ) and capacities. The $90 \mathrm{kV}$ line is unable to carry the whole load especially to Yaounde should the $225 \mathrm{kV}$ line fail. This is what makes the SIG very unreliable. The situation is better (but perhaps only marginally so) for Douala because although there are lines to Logbaba (a $225 \mathrm{kV}$ line from Songloulou, a $225 \mathrm{kV}$ line from Mangombe, and a $90 \mathrm{kV}$ line from Edea), the components are old, interconnection transformers are old and/or overloaded, and one of the two 


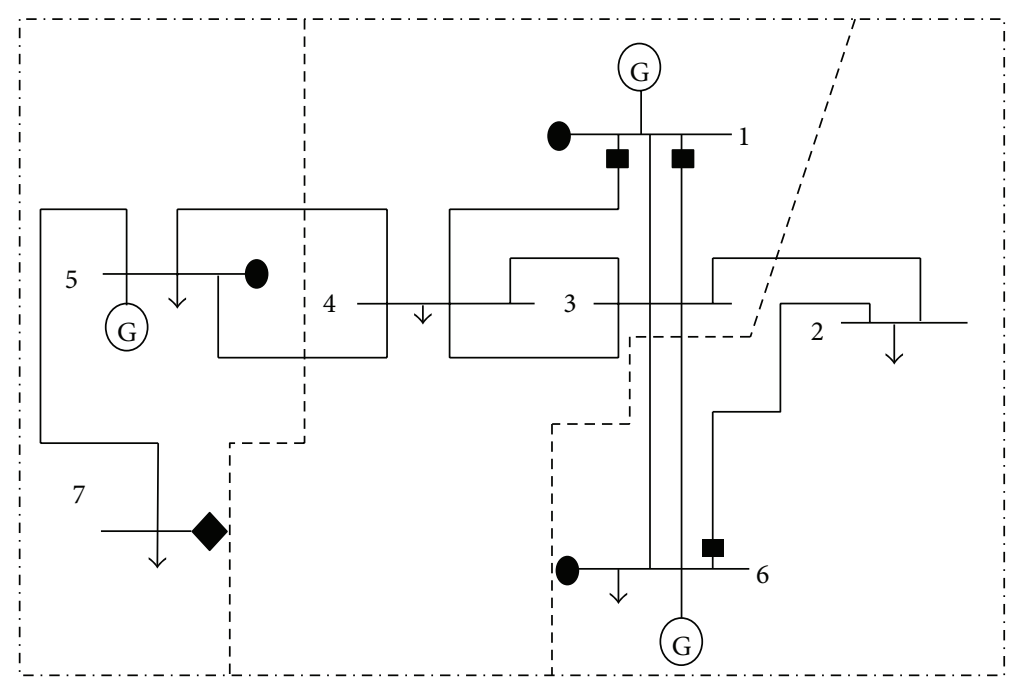
(1) Songloulou
(5) Bekoko
- Busbar voltage measurement
(2) Oyomabang
(6) Edea
Injection current measureme
Line current measurement
(3) Mangombe
(7) Nkongsamba

(4) Logbaba

FIgURE 8: Optimal placement sites for PMUs within the SIG.

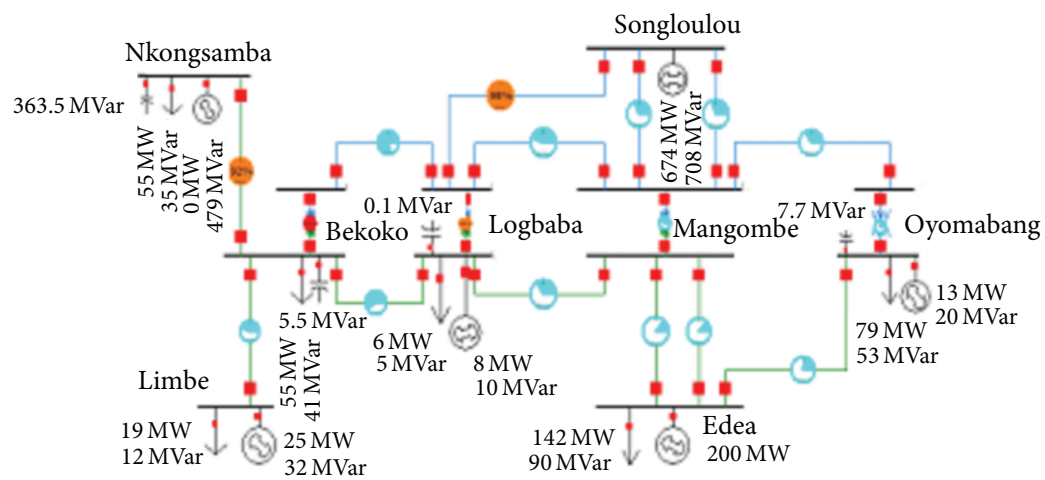

FIGURE 9: Simplified real-time model of the SIG.

$225 \mathrm{kV}$ lines is overloaded. The result of this simulation stresses the need for transmission line upgrade and renewal within the SIG [12] in order to enhance its reliability.

\section{Discussion}

Conventionally, we want the PMU placement to be optimal in the sense that it makes all buses in the system observable with a minimum number of PMUs. This is especially true for largescale complex systems [13]. The proposed algorithm is applied to the six-bus test systems under balanced conditions in which single-phase representation is adequate. This method is referred to as Madtharad's algorithm [5]. The true state of the network is computed by using power-flow calculation.

When this algorithm is applied to the SIG, starting from seven initial installation sites, it is seen that the number of placement sites has been reduced to four. The basic rule of
PMU placement is that, when a PMU is placed at a bus, it can measure the voltage phasor at that bus, as well as at the buses at the other end of all the incident lines, using the measured current phasor and the known line parameters [14]. It is assumed in this study that the PMU has a sufficient number of channels to measure the current phasors through all branches incident to the bus at which it is placed. Figure 8 illustrates the optimal placement of the PMUs within the SIG.

Other researchers, notably $[3,5,15]$, have shown that location of the PMU is important to obtain a better state estimate for a power system. Though a PMU at any location provides more accurate estimates than the case when there is no PMU, there are certain PMU locations in the network which seem to provide much higher reduction in error than at any other bus in the network. This PMU placement algorithm can therefore be followed to quickly find out the optimal location of PMUs, especially in the case of large networks. 


\section{Conclusion}

In this paper, the application algorithm for PMU measurement placement for power system state estimation is presented. The proposed algorithm finds an optimal measurement placement based on minimum condition number criterion of each subnetwork. Since the problem size is smaller, this decomposition improves the computational time required to obtain types and positions of the available measurement compared to the previously applied methods based on SCADA which consider the whole network. From the results obtained, the decomposition technique provides a promising strategy to solve the measurement placement problem of a large power system state estimation. In all cases considered [16], PMUs have a beneficial effect on the accuracy of the state estimation. Further studies will consider the dynamic model of the network.

The main contribution of this work lies in investigating the feasibility of using Madtharad's algorithm for the PMU placement problem in the Cameroon power system. Future work will include additional constraints into the PMU placement problem, such as the existence of conventional measurements, user-defined measurement redundancy at the buses, and the consideration of measurement uncertainty. These constraints are difficult to handle by conventional optimization methods. The promising results presented in this paper will encourage the researchers and utilities in using Madtharad's algorithm for the larger power systems.

\section{Glossary}

AES SONEL: The company that manages the transmission and distribution of electricity in Cameroon

Bekoko: $\quad$ Area within the city of Douala with the second largest electricity substation

Busbar: An electrical conductor onto which electric power is injected into the network or removed for distribution

Cameroon: Country in west central Africa

CAMTEL: Cameroon Telecommunications PLC

Douala: Cameroon's economic capital

Edea: City in the littoral region of Cameroon where about $40 \%$ of the country's electricity is generated

EMS: $\quad$ Energy management system

Limbe: City in the south-west region of Cameroon where the country's largest thermal (heavy fuel oil) electricity plant is located

Logbaba: Area within the city of Douala with the largest electricity substation

Mangombe: City in the littoral region of Cameroon where the electricity network load dispatch centre is located

NCC: $\quad$ Network control centre

Nkongsamba: City in the littoral region of Cameroon with a substation connecting the longest transmission lines
Oyomabang: Area within the city of Yaounde with the largest electricity substation, and some thermal plants

PMU: $\quad$ Phasor measurement unit

PowerWorld: Software used in the modeling and analysis of electric power systems

PSGuard: Power system wide area monitoring system based on $A B B$ 's process control system and Windows Server technology

Real-time: Being able to process data as it comes in SCADA: Supervisory control and data acquisition

SIG: $\quad$ Southern interconnected grid of the Cameroon power system

Songloulou: Area in the littoral region of Cameroon where about $45 \%$ of the country's electricity is generated

Substation: A branch of a main electrical power station where electric power is converted, redistributed, or modified in voltage level

Tie-line: Electricity transmission line connecting two adjacent busbars (buses) or substations

Yaounde: Cameroon's political capital.

\section{References}

[1] A. Siewe, "Communications sub-director, AES-SONEL," Cameroon Tribune, 14 pages, 2010.

[2] E. Tanyi and E. Mbinkar, "A wide area network for data acquisition and real-time control of the cameroon power system," Journal of Control Engineering and Applied Informatics, vol. 13, no. 1, pp. 5-11, 2011.

[3] A. Jain and N. R. Shivakumar, "Impact of pmu in dynamic state estimation of power systems," in Proceedings of the 40th North American Power Symposium (NAPS '08), Calgary, Canada, September 2008, report no. IIIT/TR/2009/50.

[4] D. M. Falcao, F. F. Wu, and L. Murphy, "Parallel and distributed state estimation," IEEE Transactions on Power Systems, vol. 10, no. 2, pp. 724-730, 1995.

[5] C. Rakpenthai, S. Premrudeepreechacharn, S. Uatrongjit, and N. R. Watson, "Measurement placement for power system state estimation using decomposition technique," Electric Power Systems Research, vol. 75, no. 1, pp. 41-49, 2005.

[6] F. G. Stremler, Introduction to Communication Systems, Addison-Wesley, Reading, Mass, USA, 1992.

[7] A. G. Phadke, "Synchronized phasor measurements in power systems," IEEE Computer Applications in Power, vol. 6, no. 2, pp. $10-15,1993$.

[8] E. Karam, Implementation and simulation of communication network for wide area monitoring and control systems in OPNET [M.S. thesis], OPNET, Stockholm, Sweden, 2008.

[9] ABB, Wide Area Measurement, Monitoring, Protection and Control, Industrial IT for Energy System Operation, Utility Automation Systems, ABB, Zurich, Switzerland, 2003, http:// www.abb.com/substationautomation/.

[10] E. Tonye, E-Gouvernance-Présentation Générale, Séminaire d'Information du Publique sur les Produits et Technologies Microsoft, Ecole Nationale Supérieure Polytechnique Yaoundé, 2010. 
[11] C. Madtharad, S. Premrudeepreechacharn, N. R. Watson, and D. Saenrak, "Measurement placement method for power system state estimation: part I," in Proceedings of the IEEE Power Engineering Society General Meeting, pp. 1629-1632, July 2003.

[12] J. M. Ngundam, Power Sector Development Plan 2005-2030, Transmission, Distribution and Capacity Issues (PDSE2030), Ministry of Energy and Water Resources, Yaoundé, Cameroon, 2006.

[13] J. Zhang, G. Welch, G. Bishop, and Z. Huang, "Optimal PMU pacement evaluation for power system dynamic state estimation," in Pacific Northwest National Laboratory Review, pp. 27599-23175, University of North Carolina at Chapel Hill, Chapel Hill, NC, USA, 2010.

[14] S. Chakrabarti, G. K. Venayagamoorthy, and E. Kyriakides, PMU Placement for Power System Observability Using Binary Particle Swarm Optimization, University of Cyprus Review, Nicosia, Cyprus, 2006.

[15] A. Abur, "Distributed state estimation for MEGA grids," in Proceedings of the 15th Power Systems Computation Conference (PSCC '05), pp. 22-26, Liege, Belgium, August 2005, session 5, paper 1 .

[16] M. Hurtgen and J. C. Maun, "Advantages of power system state estimation using Phasor Measurement Units," in Proceedings of the 16th Power Systems Computation Conference (PSCC '08), Glasgow, Scotland, July 2008. 

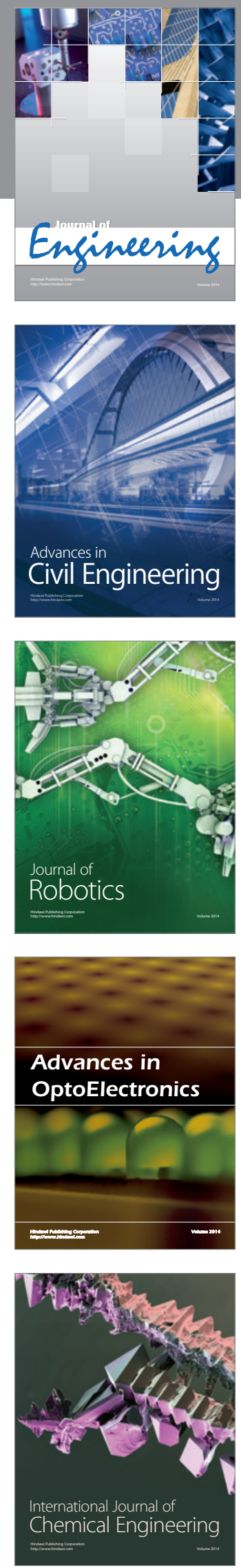

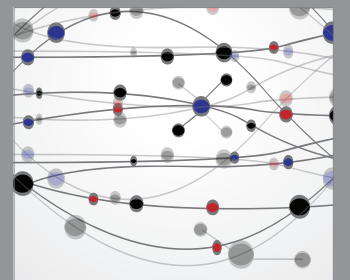

The Scientific World Journal
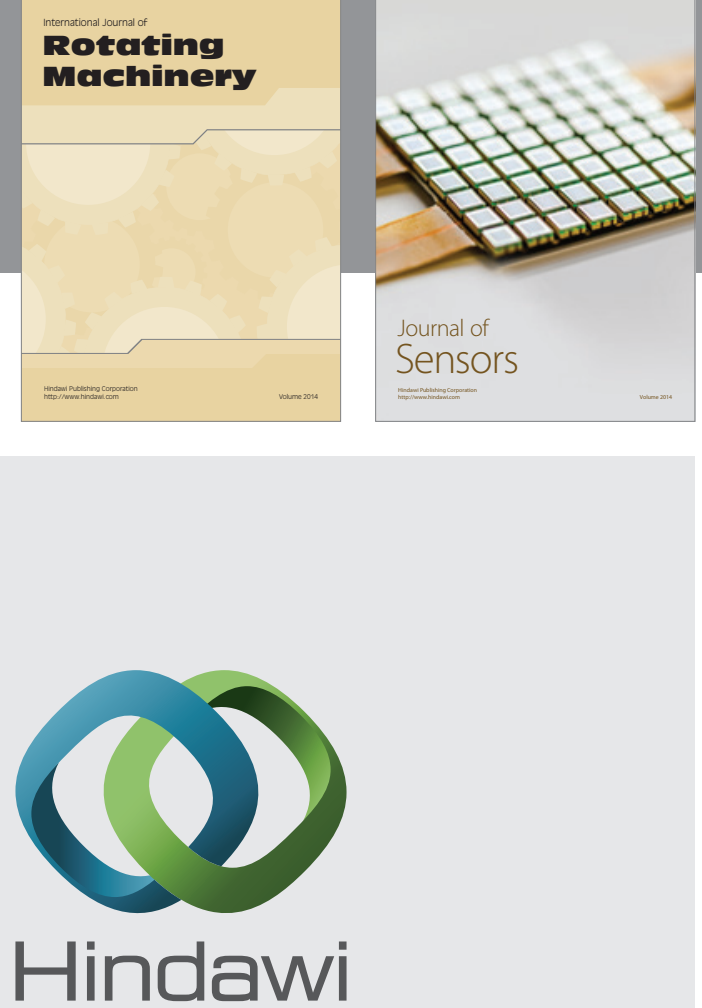

Submit your manuscripts at http://www.hindawi.com
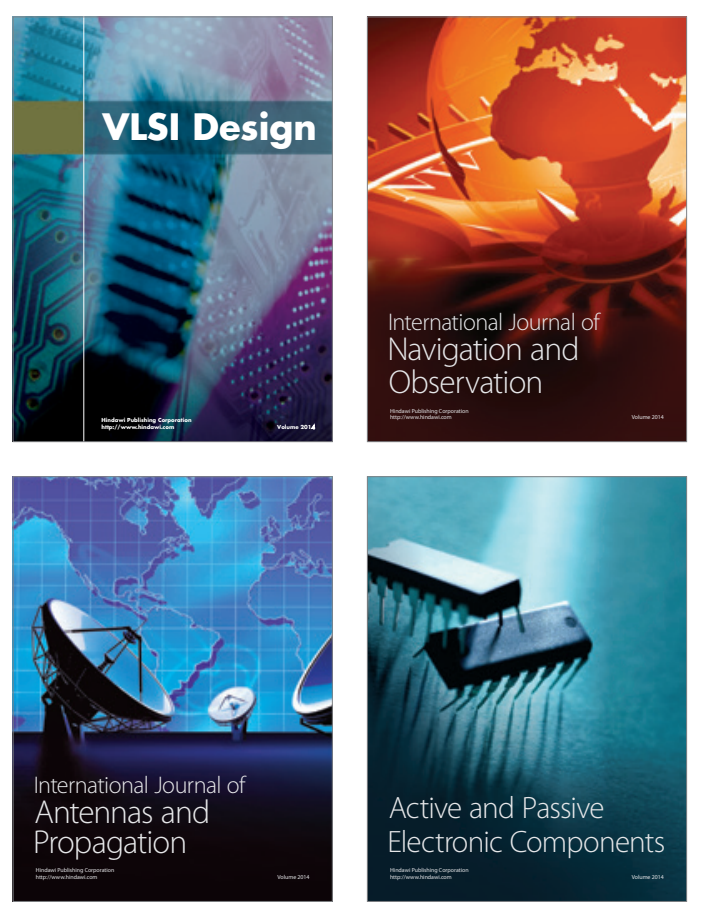
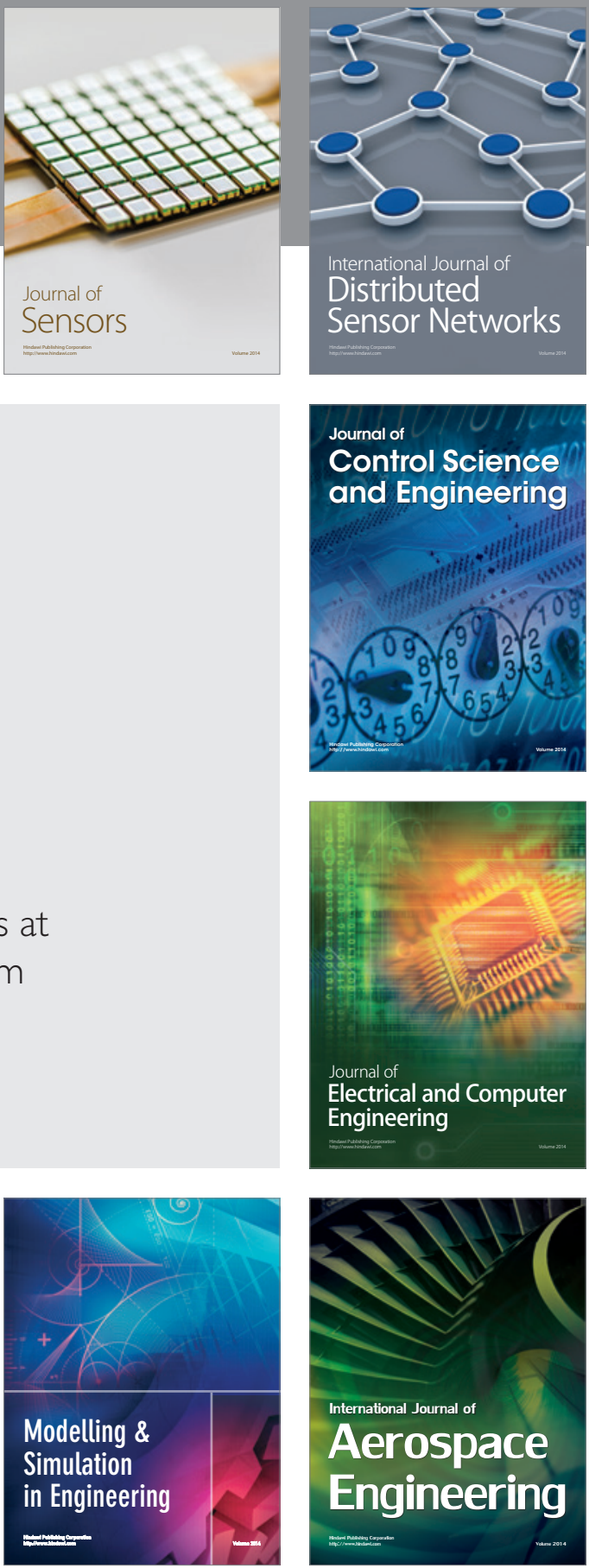

Journal of

Control Science

and Engineering
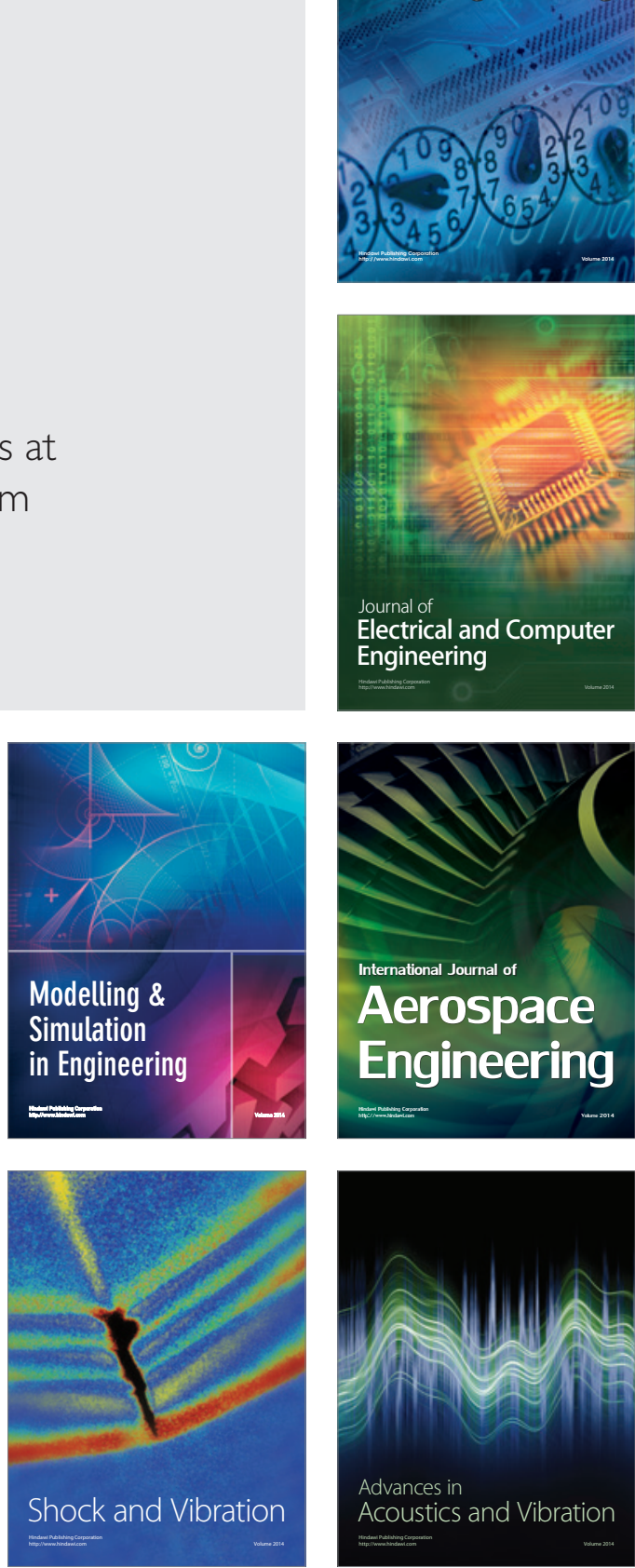